\section{Synchrotron Radiation Facilities}

Particularly in the past few years, interest in using the synchrotron radiation emanating from high energy, circular electron machines has grown considerably. In our February issue we included an article on the synchrotron radiation facility at Frascati. This month we are spreading the net wider - saying something about the properties of the radiation, listing the centres where synchrotron radiation facilities exist, adding a brief description of three of them and mentioning areas of physics in which the facilities are used.

Inevitably, an electron forced to follow a curved trajectory will emit radiation. For relativistic electrons, those travelling close to the speed of light, the total power radiated per revolution in a circular machine is proportional to the fourth power of the energy and inversely proportional to the radius of the machine $\left(E^{4} / R\right)$. This is a major problem in attempting to push the peak energy of electron synchrotrons or storage rings higher because the machine's r.f. accelerating system has to put back all the radiated energy. However it is an ill wind that blows no-one any good ... the synchrotron radiation has features which makes it an excellent experimental tool for many applications.

\section{Properties of the radiation}

A particular property of synchrotron radiation from high energy machines, which gives an additional useful handle in the understanding of some experiments, is that it is emitted with a high degree of polarization ( $100 \%$ on the plane of the synchrotron orbit and high close to the plane). Also, due to the relativistic effect, all the radiation is strongly forward focused emerging in a very narrow cone tangent to the beam.

The radiation is a continuum covering a wide spectrum of energies (usable intensities extending from $10^{-1}$ to over $10^{3}$ angströms are obtained at existing machines). The wavelength at which peak intensity occurs is reduced with increasing accelerator energy as is illustrated in the curves recorded at the DESY synchrotron shown in the figure. Note that the spectrum is like that of a 'blackbody' but peak radiated intensities correspond to blackbody temperatures of well over a million degrees. Only very high temperature plasmas or nuclear explosions provide comparable terrestrial radiation sources and the later is not a particularly practical experimental technique.

Synchrotron radiation is especially useful in the wavelength range from about 1 to 1000 angströms where it covers the gap between X-ray levels and short wavelength transmission limits.

The radiated intensities depend on such things as the accelerated electron beam intensity and, of course the electron beam energy. In the synchrotron therefore the emitted radiation pattern follows the accelerator cycle. Storage rings have the advantage of a constant energy and virtually constant intensity. Apart from being affected by such accelerator parameters, however, the physicist using synchrotron radiation can 'parasite' on a high energy particle physics programme without disturbing it or being disturbed by it.

\section{List of Synchrotron Radiation Facilities}

The following list of research centres where synchrotron radiation facilities are in action, or planned, will indicate how interest in this field has blossomed from virtually nothing about five years ago. At the electron synchrotrons: DESY, using the $7.5 \mathrm{GeV}$ machine, has led the way being the first in time and the first in quantity
Below: Variation in power radiated by an individual electron over a range of wavelengths as the energy of the accelerated beam in the synchrotron is changed. These particular curves are from the DESY machine.

Right: Diagram of the radiation facility at the DESY $7.5 \mathrm{GeV}$ electron machine where some of the leading work in this field has been carried out. The indications are : EO - electron orbit, CH-chopper, M 1-3-mirrors, B 1-2 - beam shutters, $X$-DIFF - X-ray diffractometer, UHV S - UHV sample chamber, $S$ - sample, FM - focusing mirror, $R$ 1-3 Rowland spectographs, $P G-D E S Y$ spectograph, $X-M O N O$ - X-ray monochrometer, S M-VAP - metal vapours, W 1-4 - Wadsworth monochromator.

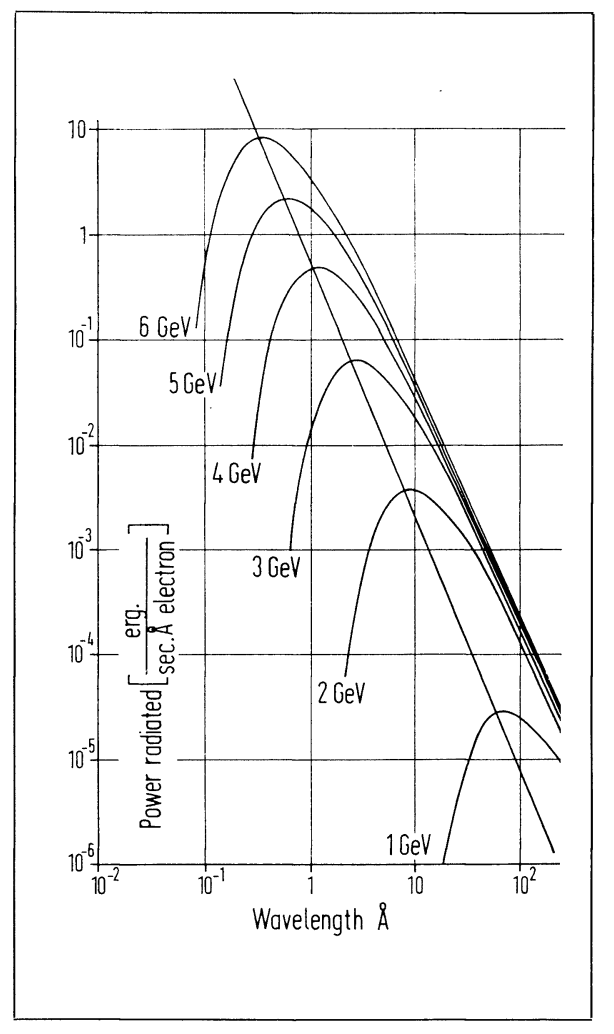

and quality of experimental results (more information below). Daresbury, using the $5 \mathrm{GeV}$ NINA, are currently bringing a new facility into action (more information below). Bonn with the 2.3 GeV machine have a small synchrotron radiation programme. Tokyo INS have one at their $1.3 \mathrm{GeV}$ machine. The Frascati facility at the 1.1 GeV machine was reported in the February issue (page 42). There are also programmes at smaller machines such as the $330 \mathrm{MeV}$ at Glasgow and the $180 \mathrm{MeV}$ at the National Bureau of Standards, USA. At storage rings: The pioneering work has been done at the $240 \mathrm{MeV}$ ring (known as Tantalus) of the Physical Sciences Laboratory, Wisconsin, which has run a programme of synchrotron radiation research for several years. There are also plans for facilities on storage rings at ACO (Orsay), the Lebedev Institute (Moscow) and a large one on DORIS (DESY). 




The drawing shows the scope of the existing facility on the DESY synchrotron and the instruments currently in use. The facility is divided into two floors (the upper floor coming into full operation in 1971), the beams being split by mirrors, and there is a further division on the lower level so that several experiments can run simultaneously. The facility has been used almost from the time that the synchrotron came into action and a high proportion of the papers resulting from research at the machine have involved the use of synchrotron radiation. In 1971 there were 25 scientists (from DESY, Hamburg, Munich and Freiburg) working full time at the facility as well as part-time visitors from Heidelberg, Helsinki and Sendai.

$A$ second synchrotron radiation laboratory is being built at the DESY machine for the European Molecular Biology Organization, EMBO. The building itself is complete and installation of the beam pipe, detectors etc. has begun. In addition, a facility will be established at the electron-positron storage rings, DORIS, scheduled for operation at the end of 1973.

The facility at Daresbury is now being commissioned. It is divided into three sections - two areas (operationally independent) each fed by a beam-line and a control area where a Honeywell 316 computer is stationed with a fast data link to the Laboratory central computer (IBM 360/65). There are also three independent branch highways in CAMAC whereby the H 316 provides interactive links between the data collected and the apparatus.

Instruments include a horizontally dispersing Wadsworth monochrometer (covering the range 500 to 3500 angströms with a resolution of a few angströms), a grazing incidence monochrometer (40 to 350 angströms with a resolution better than 1 angström) and a vertically dispersing monochrometer which should be able to exploit the polarization property of the radiation (400 to 5000 angströms with a resolution better than 0.5 angström). The experimental programme will involve scientists from Cambridge, Daresbury, Manchester, Medical Research Centre, National Physical Lab., Oxford and Reading.

The facility at the PSL Wisconsin storage ring is, at present, the most extensive in terms of the number of beams (six outlet ports) and experimental groups (fourteen in 1971) that can be supported. The total number of groups using or intending to use the facility is now 25. PSL was also the venue for a 'Synchrotron Radiation Users Group Conference' in November 1971.

This year the National Science Foundation is funding the construction of two additional beam-lines and the
University of Wisconsin has approved the building of additional experimental area (to provide four times the previous area) so that the new beam-lines can be exploited and the existing beam-lines extended. The shutdown for the modifications is planned for early Autumn. It is hoped that a microtron will then be installed as injector (microtrons can give lower emittance and energy spread than the FFAG machine used at present).

Operation of the storage ring as a radiation facility has proved very efficient; only $2 \%$ of scheduled research time was lost due to equipment failures in 1971. Three computers (PDP 8e, PDP 12 and IBM 1401) are in use for data acquisition and some control functions. Experimental equipment includes a Pruett-Lien normal incidence monochrometer which was commissioned in 1971 and several other monochrometers. A wavelength shifter will be installed during the shutdown.

An improved version of the storage rings is under study (Tantalus II) to make research at shorter wavelengths possible. The peak energy of the new ring would be $1 \mathrm{GeV}$; it would be fed by a $35 \mathrm{MeV}$ microtron. Such a ring would radiate a continuum from infrared to the X-ray region with a peak around 10 angströms and with radiated intensities generally several orders of magnitude higher than from Tantalus I. 